\title{
1 Virtual Reality Balance Games Provide Little Muscular 2 Challenge to Prevent Muscle Weakness in healthy 3 older adults
}

5 Dr. Aijse W. de Vries ${ }^{\mathrm{a}}$, Jente Willaert (joint first author) ${ }^{\mathrm{b}}$, Prof. Dr. Ilse Jonkers ${ }^{\mathrm{b}}$, Prof. dr. Jaap H. van

6 Dieën ${ }^{\mathrm{c}}$, Prof. dr. Sabine M.P. Verschueren ${ }^{\mathrm{a}}$

$7 \quad{ }^{a}$ Musculoskeletal Research Unit, Department of Rehabilitation Sciences, Faculty of Kinesiology and

8 Rehabilitation Sciences, Katholieke Universiteit Leuven, Leuven, Belgium

$9 \quad{ }^{b}$ Human Movement Biomechanics Research Group, Department of Kinesiology, Faculty of Kinesiology

10 and Rehabilitation Sciences, Katholieke Universiteit Leuven, Leuven, Belgium

$11{ }^{\mathrm{c}}$ MOVE Research Institute Amsterdam, Faculty of Human Movement Sciences, VU University

12 Amsterdam, Amsterdam, the Netherlands

14 Corresponding author: Prof. Dr. Sabine Verschueren 


\section{Abstract}

26 Objective: Muscle weakness is an important risk factor for falls in older adults. Intensity and duration of 27 muscle activity are important determinants of exercise effectiveness in combating muscle weakness. The 28 aim of this paper was to assess the intensity and duration of muscle activity in Virtual Reality (VR) 29 balance games.

30 Materials and Methods: Thirty young and thirty healthy older adults played seven different VR balance

31 games. Muscle activity of the Vastus Lateralis, Vastus Medialis, Soleus and Gluteus Medius was obtained 32 using surface EMG. The processed EMG signals were divided in $200 \mathrm{~ms}$ blocks, after which each block was 33 categorized by its average normalized EMG activity i.e. $>80 \%, 60-80 \%, 40-60 \%$ or $<40 \%$ of maximum 34 voluntary contraction (MVC). We calculated the total number of blocks in each category to score 35 intensity, as well as the maximal number of consecutive blocks (MCB) $>40 \%$ MVC, to identify prolonged 36 muscle activity.

37 Results: Muscle activity during game-play was mostly below 40\% MVC and prolonged activation was

38 lacking. Only the games that included more dynamic movements showed activation blocks of higher 39 intensity and resulted in more MCB.

40 Conclusion: Our method allowed us to analyze the overall muscle activity and the distribution of activity 41 over a trial. Although the activation levels during these VR games were low in general, we identified 42 game elements that could potentially provide a strength training stimulus. Future research should aim to 43 implement these elements, such that the intensity, prolonged activity and rest are optimized to 44 sufficiently challenge lower limb muscles in VR training. 
Age-related decreases in muscle strength contribute to the increased incidence of falls at higher age ${ }^{1,2}$. Muscle strength of the lower limbs has been shown to be a predictor of functional balance ${ }^{3}$, postural stability ${ }^{4}$ and fall incidence in older adults ${ }^{1,5,6}$. Fortunately, sufficiently challenging exercises combined with optimized nutrition provides similar muscle protein synthesis in older compared to young adults ${ }^{7}$. Indeed, older adults can improve muscle strength and power after resistance training ${ }^{7,8}$.

53 Virtual reality training (VRT), an alternative training method that combines playing games using 54 commercial consoles (e.g. Wii console, Kinect) and exercising, is applied to improve balance in older adults $^{9,10}$. The term virtual reality (VR) covers a wide range of applications as described in the virtuality reality continuum ${ }^{11}$. In this study, VR is defined as computer games using commercial consoles as Wii and the Kinect console and that are controlled with body movements. VRT might lead to increased motivation because of the gaming element ${ }^{12,13}$, which could help against low adherence in home-based training programs ${ }^{14,15}$. Furthermore, VRT creates a training environment in which either direct (e.g. coach on the screen gives feedback on the posture of the player) or indirect feedback (e.g. scoring points of the game) can be provided. Feedback can help to increase physical activity during the game ${ }^{16}$. However, systematic reviews yielded conflicting results on the fact whether VRT can improve (components of) balance in elderly 9,17,18. This might be explained by the wide variety in VRT-tools and the different nature of the games. For VR to have an optimal effect on biomechanical constraints of balance ${ }^{4}$, it should challenge balance, by going to the individual limits of stability ${ }^{4,19,20}$, and also include muscle strength training ${ }^{21,22}$. However, all these goals have to be pursued keeping in mind the person's safety. Effects of training programs on muscle strength, as a determinant of balance, have received limited attention ${ }^{23}$ and the muscular demands of different VRT programs have not been studied.

Training load is affected by overall muscle effort, which depends on the relative intensity of muscle activity and the amount of rest between periods with substantial muscle activity ${ }^{24}$. Recommendations regarding conventional strength training in older adults suggest using moderate to heavy intensities, i.e., $>60 \%$ of the maximum weight that can be moved for one repetition ${ }^{25}$. Strength training using higher velocity concentric exercises improves muscle power and is more effective than traditional strength

74 training in improving functional performance, as reflected in sit to stand, jump height and reaction 75 time ${ }^{26}$. Furthermore, the rate of torque development appears to help discriminate fallers form non fallers ${ }^{27}$, suggesting that muscle strength should also be trained at high velocities. 
77 Weight bearing exercises, possibly with added extra weights, are more suited for VRT than externally

78 loaded exercises using weight machines. However, muscle activity during weight bearing exercises might

79 be low. To maximize muscle fiber recruitment, it is recommended to add steps or to increase the number

80 of repetitions until muscle exhaustion ${ }^{8,28}$. The introduction of rests within a training set has been shown

81 to attenuate increases in growth-hormone release and strength gains following a training regimen ${ }^{29}$.

82 Therefore, it is important to also consider the duration of the bouts of muscle activity within a training

83 session. High intensity exercises at high speed will induce muscle hypertrophy, however a high number

84 of repetitions at lower intensities will induce muscle fatigue and as such also induce muscle hypertrophy

85 . Low intensities are potentially more suitable for elderly compared to high intensities since these

86 exercises are more accessible, safer and pleasant to perform ${ }^{30}$.

87 The aim of this study was therefore to assess intensity and duration of lower limb muscle activation

88 elicited by different commercially available VRT applications in young and older adults. The results were

89 interpreted in the context of reported recommendations for conventional strength training $8,25,31$.

\section{Methods}

91 Participants

92 Table 1. Subject characteristics.

93 Thirty young and thirty healthy older adults (aged $>65$ ) were recruited using flyers around the sports

94 facilities of the KULeuven (Table 1). All participants had to be free of any pathology of the

95 musculoskeletal and neurological or vestibular system, cardiovascular disease or diabetes that would

96 prevent them from safely performing exercises. Participants signed written informed consent prior to

97 participation, in accordance with the Declaration of Helsinki. The study was approved by the local ethics

98 committee (Commissie Medische Ethiek K.U. Leuven). All participants completed the Mini Mental State

99 Examination (MMSE) to ensure they were in a cognitive good state. Participants had to score at least 25

100 out of 30 to be eligible for the study. Mean score for the test was 29.3 and no participants were excluded

101 (Table 1).

102 Material

103 Seven games involving different movements were tested (Table 2 and appendix for full description of the

104 games). Games were selected on their potential ability to challenge balance or muscle activity or were

105 used in VR balance training studies previously ${ }^{10,18,32}$. Games were selected to represent a varied but 
realistic sample of games that can be used as VR training at home or in retirement homes and small rehabilitation facilities. There are different forms of virtual environments that can be used for VRT. In this study, we will focus on projection-based environments ${ }^{33}$. The games were controlled by the Xbox Kinect camera (Microsoft, US), which tracks the players' movements via depth sensing cameras, or by the Wii Balance Board (Nintendo, Japan), which registers movements of the Center of Pressure (COP). Recommendations regarding conventional strength training in older adults suggest using $>60 \%$ of the maximum weight that can be moved for one repetition (1RM) ${ }^{25}$. However, this $1 R M$ concept cannot be used for unloaded exercises. Therefore, the intensity in unloaded exercises is hard to quantify and we used surface electromyography (EMG) to measure muscle activation. EMG signals that are normalized to values achieved during maximum voluntary contractions (MVC) have shown to be approximately linearly correlated with force ${ }^{34,35}$. Neuromuscular activation was obtained using silver-silver chloride, pre-gelled bipolar electrodes (Ambu Blue Sensor, DK) connected to an eight-channel wireless EMG system (Aurion, Zero-wire, IT), which was recorded in Nexus (Vicon, Oxford Metrics, UK) at $1000 \mathrm{~Hz}$.

Table 2. Overview of the included games.

Before participants engaged in the VRT experiments, EMG electrodes were placed on four lower limb muscles which have been shown to be important in balance and preventing falls in older adults ${ }^{1,36,37}$; Vastus lateralis (VL), Vastus medialis (VM), Soleus (Sol) and Gluteus medius (GluM). For practical reasons, we chose to measure muscle activity unilaterally. Electrodes were consistently placed on the left leg, according to the SENIAM guidelines ${ }^{38}$. Maximal voluntary contraction (MVC) was obtained according to SENIAM guidelines ${ }^{38}$. Participants played seven different games in a randomized order, during which EMG signals were recorded. The shorter games (Adventure, Wiiski, Kinski, Wiiyogaw, Wiiyogahm), were played three times, whereas the longer games (Boxing and Kinyoga) with repeating sequences were played once. After each game, the participants were asked to sit down and take a rest for approximately

130 five minutes.

\section{Processing}

132 All processing was performed in Matlab (MathWorks, USA). EMG signals were high-pass filtered at 20Hz, 133 using a 3rd order high-pass Butterworth filter before being rectified and smoothed using a moving 134 average technique with a window of $100 \mathrm{~ms}^{39}$. The processed signals from the experimental trials were normalized to maximum values obtained during the MVCs. 
137 Traditional outcome measures quantifying intensity obtained from EMG-data include the area under the curve, mean, median or maximum values ${ }^{40}$. However, these methods lose potentially valuable temporal and amplitude information. Therefore, muscle activity was averaged over 200-ms blocks and categorized 140 each block in one of four activation zones, $<40 \%, 40-60 \%, 60-80 \%$ and $>80 \%$ MVC (fig. 1). The number of

141 blocks in each zone was counted and total time spent in each zone was normalized to the duration of the 142 game, to express the Time in Zone (TIZ). This normalization was done because a shorter game will more 143 likely result in less blocks than longer games, but could in practice be played several times to 144 accommodate for this difference. Larger metabolic stress is expected from a high number of consecutive 145 blocks of muscle activation and plays a crucial role in strength gains following exercise at lower 146 intensities ${ }^{29}$. Therefore, we calculated the maximal number of consecutive 200-ms blocks (MCB) 147 separated by no more than three seconds of $<40 \%$ MVC EMG activity.

148 Figure 1, Example of blocks detection: The smoothed EMG amplitude of the VL muscle during a section of the 149 Adventure game is plotted in grey. The colored 200-ms blocks represent different activation zones, based on the 150 average of the smoothed signal over these 200-ms blocks.

151 Statistics

152 The games that provoke very low muscle activation were omitted from further statistical analysis.

153 Treshold was defined as less than $5 \%$ of total game duration activations higher than $40 \%$ of MVC and in 154 addition a low number of MCB's. Generalized Estimating Equations (GEE), were used to perform null 155 hypothesis testing for the factors game and age group on the variables TIZ and MCB for all muscles, with 156 trial number as a covariate. The correlation structure was set to autoregressive. Post-hoc pairwise 157 comparisons were done using Least Significant Difference (LSD). No previous effect sizes were available 158 to estimate power and sample size. Since our main focus was to test the proof of concept and feasibility 159 of the novel exergames, only a limited sample size of 16 subjects were recruited. All statistical analysis 160 were performed in IBM SPSS Statistics Version 21.0.

\section{Results}

162 Wiiyogaw, Wiiyogahm and Wiiski, all using a COP-based controller, provoked only minor muscle 163 activation parameters and were omitted from further statistical analysis. 
In general, all games elicited during less than $20 \%$ of total game duration a muscle activation higher than $40 \%$ of the MVC. The longest periods of consecutive high muscle activity were very short $(<5 \%)$ in almost all games. The number of MCB that muscles were active at or above $40 \%$ of the MVC was also low.

Time in Zone

To assess duration of lower limb muscle activation, TIZ for every activity zone was calculated. TIZ was compared between age groups and games (Fig 2, Table 3), and in case of an interaction effect further analyses were performed (Fig 3).

For older adults, a very low time $(<5 \%)$ with activations higher than $40 \%$, was seen for the GluM in all games. However, for the $>40 \%$ categories Adventure showed longer periods of activation compared to the other games.

For the VM and VL, longer TIZ was seen for the adventure game compared to the other games for all activation zones except the lowest activation zone. For the latter, the Kinski game showed the longest time.

For the SOL, longer activation in the $>60 \%$ activation zones was seen for both Adventure and Boxing game compared to the other games.

Table 3. TIZ: Summary of the GEE model effects.

Figure 2. TIZ descriptive statistics. In rows, games, muscles and age groups ( $\mathrm{y}=\mathrm{young}$, e=elderly) are indicated. Along the $\mathrm{x}$-axis the TIZ as a percentage of the total game duration is plotted. The TIZ for the four different categories are stacked, to provide an overview of the total time that each muscle is active during each game.

\section{Figure 3. TIZ post hoc comparisons}

Panels show mean values and standard deviations of $\mathrm{TIZ}$, horizontally grouped by muscle and vertically grouped by activity zone. Significant differences $(P<.05)$ between games are marked ' $x$ ' spanning groups, and in case game $x$ group interactions were found and between game comparisons were made within the elderly group ' ${ }^{*}$. Significant group differences within games are indicated ' $\#$ '.

$a d v=$ Adventure, kiny= Kinyoga, box= Boxing, kski= Kinect ski

\section{Between-game differences}

\section{Group differences}

For the $\mathbf{V L}$, during Adventure, young participants showed longer TIZ in the $>80 \%$ category than older adults. In contrast, during the Boxing game older adults spent more time in the $>80 \%$ category. In the 40 $60 \%$ category, older adults had longer TIZ than young participants across all games. Furthermore, young 
participants had longer TIZ in the $<40 \%$ category during both Boxing and Kinyoga.

The VM did not show a significant game*age effect. However, a significant main effect of age indicates that older adults show slightly more activity than young in the $40-60 \%$ across all games.

For the SOL, a significant game*age effect was seen for all activation zones. Young participants showed longer TIZ in the $>60 \%$ categories than older adults during Adventure. In the $40-60 \%$ category, older adults had longer TIZ than young subjects during, Kinski, Boxing and Kinyoga. In the lowest category, older adults had longer TIZ than young during Adventure whereas young participants spent more time in this category during Kinyoga.

For the GluM, in the $40-60 \%$ category, older adults showed longer TIZ than young participants during Kinski, Boxing and Kinyoga. In the lowest activity category, young subjects had longer TIZ during the Boxing and Kinyoga game.

\section{Maximal Consecutive Blocks}

Larger metabolic stress is expected from a high number of consecutive blocks of muscle activation and plays a crucial role in strength gains following exercise at lower intensities ${ }^{29}$. For this, we calculated the MCB for each muscle in the Adventure, Kinyoga and Boxing game (Table 4).

Table 4. GEE model (game, age and their interaction) effects for MCB for VL, VM, SOL and GluM. . (>40\%MVC) (fig 4). It should be noted that the median values of the games that elicited the longest active periods, indicate that the muscle was active for a consecutive bout of only 8 seconds. For all muscles a game*age interaction was found for the MCB.

Figure 4. Maximal consecutive blocks 
225 VL and VM showed higher MCB, reflecting more consecutive muscle activity, during Kinyoga than during

226 Adventure and Boxing in older adults. SOL and GluM did not show significant differences between

227 games.

228 Group differences

229 For the VL, SOL and GluM, older adults showed more MCB than young subjects during Kinyoga (and

230 Boxing for the GluM). In the SOL, young participants showed more MCB than older participants during

231 Adventure. For the VM, there were no group differences.

\section{Discussion}

233 The aim of this study was to identify potential effective VR games for muscle strength training. Muscle

234 strength training is seen as one of the balance components that need training in elderly. The percentage

235 of total game-time that a muscle was active in pre-defined activity categories was studied, as well as the

236 MCB that muscles remained active, were studied. Both factors are seen as indicators of overall muscular

237 effort during different VRT-games.

238 In general, all included games elicited very low muscle activity. Most of the games elicited substantial 239 muscle activity (> 40\% of MVC) only during a small fraction of the total game-time. The longest periods

240 of consecutive muscle activity at more than 40\% MVC were very short. Especially GluM activation was

241 most of the time in the $<40 \%$ MVC category. Regarding the MCB that muscles were active at or above

$24240 \%$ was also low. This raises questions on the effectiveness of these games in improving muscle

243 strength.

244 Only few studies investigated muscle activation during exergames, and used a different approach to 245 assess this activation. The study of Soltani et al., where EMG of the upper limb was recorded during a 246 swimming exergame ${ }^{41}$, reported muscle activations in the arms between 4.9 and $95.2 \%$ of the MVC.

247 Further, Da Silva et al reported low activation (relative to the maximum voluntary contraction) of the 248 quadriceps during Wii games, which is in line with the low activations we found for the Wii games ${ }^{42}$.

249 None of these studies used such an extensive approach as the present one (i.e. by using the novel 250 activation measures; time in zone and maximal consecutive blocks) to determine muscle activation. 251 Game differences 
For all muscles, the Adventure game elicited the highest percentage of game time at muscle activity above $80 \%$. This highly dynamic game, with frequent squatting and jumping movements, also had the

254 highest percentages of activation in the 60-80\% category. The Kinski game, with frequent weight shifts

255 but only occasional jumps, resulted in the lowest TIZ in the higher activity zones. Consequently, muscle 256 activation was more prominent in the $<40 \%$ MVC category during Kinski for VL, VM and Sol. For the 257 GluM, Kinyoga and Kinski did not differ in this category. The longer TIZ in the $<40 \%$ category during the 258 Kinski game, reflects that the combined TIZ of the higher categories is longer during Adventure, Kinyoga 259 and Boxing. The Kinyoga and Boxing games incorporated dynamic exercises (e.g. squats, lunches and one 260 leg stance), but these were performed at lower speeds than in the Adventure game.

261 The games that scored lowest on muscle activation parameters (Wiiyogaw, Wiiyogahm and Wiiski) were 262 omitted from statistical analysis. It is interesting to note that the Kinski game seemed favorable over the 263 Wiiski game when looking at the muscle activation despite the similar objective. This difference may be 264 attributable to the different controller, kinematic controller (Kinski) and force plate (WiiSki). A kinematic 265 controller, which tracks kinematics, may impose more dynamic and varied movements, such as jumps, 266 squats and taking steps. This is in agreement with findings of our previous study, that games with 267 different type of controllers, lead to different center of mass displacements ${ }^{43}$.

The MCB measure was introduced to study the effectiveness of the game in inducing a prolonged muscle training stimulus at more than $40 \% \mathrm{MVC}$, to induce the desired metabolic stress ${ }^{29}$. The variations in duration, from around one minute for the adventure and the skiing games up to more than seven minutes for Kinyoga, might obscure the effectiveness of the longer games, when only evaluating the TIZ measure. Indeed, we found that for the VL and VM muscles, the Kinyoga game, which incorporated

273 structured sets of squats at a low pace, induced a higher number of MCB than Adventure, which 274 performed better for the TIZ measure. However, the number of MCB and therefore the duration of 275 sustained muscle activity was still low. In the future, research can be conducted with focus on the 276 development of games with fast and/or specific movements (e.g. squatting, single leg stance), adding 277 extra weights (weight belts) or adding steps to increase muscle activation.

\section{Group differences}

279 Differences in muscle activation level and duration between young and older subjects were found. For 280 the VL young subjects showed longer TIZ in the $>80 \%$ category during the Adventure game, whereas 281 older subjects showed longer TIZ in the Boxing game. For Sol, young subjects had longer TIZ during the 282 Adventure in both the $>80 \%$ and $60-80 \%$ categories, and during Kinski in the $>80 \%$ category. These 
differences might be attributable to the incentive that is used to elicit more movements. In both the Adventure and Kinski game, the player is encouraged to squat and jump to speed up the game. However, older adults are typically less inclined than young to speed up these games. Furthermore, the jumps might be outside of the comfort zone of the older players, resulting in lower TIZ for the $>80 \%$ category. The Boxing and Kinyoga game on the other hand, have coaches that perform the movements that the player has to follow and the player is rewarded more points when performing the movements in time. This type of game dictates the speed at which the movements are performed, and might therefore be more challenging for older adults. The caveat in a game design with fixed difficulty would be an inappropriate difficulty level, which could result in negative feedback and decrease motivation and effectiveness ${ }^{44,45}$.

\section{Limitations}

The multitude of comparisons, with significant, but small, differences between age groups and games, could obscure the main finding, i.e. that all included games elicited very low muscle activity, especially the games omitted from the statistical analysis. However, since the exact criteria at which muscle activation during weight bearing exercises can be effective are not well established, we choose to study multiple outcome measures. Nonetheless, the main finding is that in all studied VRT, the muscle activity appears low.

For traditional strength training, recommendations are to use at least $60 \%$ of $1 \mathrm{RM}{ }^{25}$, but for functional weight-bearing exercise these thresholds are less well established. Furthermore, there is no exact linear relationship between EMG and force. However, it is shown that higher EMG correlates with higher forces. Additionally, if EMG is normalized to values achieved during MVC they are approximately linearly correlated with force ${ }^{34,35}$. Therefore, we categorized the EMG activity in different activity zones based on the level of muscle activity. Especially the faster movements induced activity in the highest (>80\% MVC) zone, which is in line with findings of Soltani et al. They find higher muscle activations in games that were played at higher compared to lower velocities ${ }^{41}$. When lower loads are used it is recommended to increase the number of repetitions up to muscle exhaustion ${ }^{8}$. However, the small amount of prolonged muscle activity, as indicated by the low MCB, shows that it is unlikely that the games studied challenge the player enough to reach muscle exhaustion. Yet there is no literature on the duration of muscle activity and the accompanying recovery time required to induce an optimal metabolic response. Three seconds without any muscle activation blocks, was chosen as the cut-off point for initiating a new series. Small changes of this threshold, ranging from one to ten seconds, did not lead to 
314 different conclusions for this study. Low muscle activity can also be due to low effort of the subject while 315 playing the games. Game performance scores were not assessed, however based on our observations it 316 seemed that participants tried their best when playing the games.

317 Data were collected and analyzed in the present study with only focus on muscle activations, as one of 318 the factors that need to be trained for balance. It is evident that other factors are also important to 319 improve balance in elderly (e.g. center of mass movement, muscle force, sensory and movement 320 strategies ...) and that the balance component that needs most training can vary between patients ${ }^{4,19}$. 321 This differentiation was beyond the scope of this manuscript.

\section{Conclusions}

323 Overall, the muscle activity when performing the VRT-games studied seems low for effective muscle 324 strength training. This means that muscular benefits for the tested games are unclear. However, the 325 games could have other beneficial effects such as on cardiovascular health or wellbeing. Muscle activity 326 was only high during jumping, indicating that certain elements of these games could be part of an 327 effective muscle training, when suited for the participants.

328 In future studies, games with higher muscle activity (e.g. more dynamics movements, kicks, ...) should be 329 developed and tested, keeping the safety of participants, i.e. elderly, in mind ${ }^{46-49}$. To improve VR-games 330 for muscle strength training, muscle activity should be higher (e.g. $>40 \%$ of the MVC) and better 331 structured over time (e.g. longer bouts), to induce higher metabolic stress and make better use of the 332 total game time. Especially increasing the length of the sets, while keeping the muscle activity at a low 333 percentage of the MVC seems a more viable way for VR training. Furthermore, the incentives to trigger 334 more challenging exercises should be adequate for the target population, and preferably be adaptable to 335 the physical fitness of the individual player.

336 In conclusion, VRT-games have potential for muscle strength training in elderly, however, in future, 337 games should be developed with the purpose to sufficiently activate and thereby train their muscles, 338 since the existing games elicit too low muscle activity

\section{Acknowledgements}

340 We thank the participants for their participation in the experiments.

\section{Author disclosure statement}

342 No competing financial interests exist 
343 Corresponding author

344 Prof. Dr. Sabine Verschueren

345 


\section{References}

347 1. Moreland JD, Richardson JA, Goldsmith $\mathrm{CH}$, Clase CM. Muscle Weakness and Falls in Older Adults: 348 A Systematic Review and Meta-Analysis. J Am Geriatr Soc. 2004;52(7):1121-1129.

$349 \quad$ doi:10.1111/j.1532-5415.2004.52310.x

350

2. Rubenstein LZ. Falls in older people: epidemiology, risk factors and strategies for prevention. Age Ageing. 2006;35(suppl 2):ii37-ii41. doi:10.1093/ageing/afl084

3. Daubney ME, Culham EG. Lower-extremity muscle force and balance performance in adults aged 65 years and older. Phys Ther. 1999;79(12):1177-1185.

4. Horak FB. Postural orientation and equilibrium : what do we need to know about neural control of balance to prevent falls ? Age Ageing. 2006:7-11. doi:10.1093/ageing/afl077

5. Cattagni T, Scaglioni G, Laroche D, Van Hoecke J, Gremeaux V, Martin A. Ankle muscle strength discriminates fallers from non-fallers. Front Aging Neurosci. 2014;6:336. doi:10.3389/fnagi.2014.00336

6. Shad BJ, Thompson JL, Breen L. Does the muscle protein synthetic response to exercise and amino acid-based nutrition diminish with advancing age ? A systematic review. 2019. doi:10.1152/ajpendo.00213.2016

7. Hess JA, Woollacott M. Effect of High-Intensity Strength-Training on Functional Measures of Balance Ability in Balance-Impaired Older Adults. J Manipulative Physiol Ther. 2005;28(8):582590. doi:http://dx.doi.org/10.1016/j.jmpt.2005.08.013

8. Van Roie E, Delecluse C, Coudyzer W, Boonen S, Bautmans I. Strength training at high versus low external resistance in older adults: effects on muscle volume, muscle strength, and force-velocity characteristics. Exp Gerontol. 2013;48(11):1351-1361. doi:10.1016/j.exger.2013.08.010

9. van Diest M, Lamoth CJC, Stegenga J, Verkerke GJ, Postema K. Exergaming for balance training of elderly: state of the art and future developments. J Neuroeng Rehabil. 2013;10:101. doi:10.1186/1743-0003-10-101

10. Donath L, Rössler R, Faude O. Effects of Virtual Reality Training (Exergaming) Compared to Alternative Exercise Training and Passive Control on Standing Balance and Functional Mobility in 
Healthy Community-Dwelling Seniors: A Meta-Analytical Review. Sport Med. 2016:1-17. doi:10.1007/s40279-016-0485-1

11. Milgram P. Augmented reality: A class of displays on the reality-virtuality continuum. Telemanipulator telepresence Technol. 1994;2351:282-292.

12. Fitzgerald D, Trakarnratanakul N, Smyth B, Caulfield B. Effects of a wobble board-based therapeutic exergaming system for balance training on dynamic postural stability and intrinsic motivation levels. J Orthop Sports Phys Ther. 2010;40(1):11-19. doi:10.2519/jospt.2010.3121

13. de Vries AW, van Dieën JH, van den Abeele V, Verschueren SMP. Understanding Motivations and Player Experiences of Older Adults in Virtual Reality Training. Games Health J. 2018. doi:10.1089/g4h.2018.0008

14. Forkan R, Pumper B, Smyth N, Wirkkala H, Ciol MA, Shumway-Cook A. Exercise adherence following physical therapy intervention in older adults with impaired balance. Phys Ther. 2006;86(3):401-410. doi:10.1046/j.1532-5415.2002.50601.x

15. Simek EM, McPhate L, Haines TP. Adherence to and efficacy of home exercise programs to prevent falls: A systematic review and meta-analysis of the impact of exercise program characteristics. Prev Med (Baltim). 2012;55(4):262-275. doi:http://dx.doi.org/10.1016/j.ypmed.2012.07.007

16. Lyons EJ. Cultivating Engagement and Enjoyment in Exergames Using Feedback, Challenge, and Rewards. 2015;4(1):12-18. doi:10.1089/g4h.2014.0072

17. Faude O, Donath L, Ro R. Effects of Virtual Reality Training ( Exergaming ) Compared to Alternative Exercise Training and Passive Control on Standing Balance and Functional Mobility in Healthy Community-Dwelling Seniors : A Meta-Analytical Review. 2016:1293-1309. doi:10.1007/s40279-016-0485-1

18. Goble DJ, Cone BL, Fling BW. Using the Wii Fit as a tool for balance assessment and neurorehabilitation: the first half decade of “Wii-search." J Neuroeng Rehabil. 2014;11(1):12-. doi:10.1186/1743-0003-11-12

19. Sherrington C, Tiedemann A, Fairhall N, Close J, Lord S. Exercise to prevent falls in older adults : an updated meta-analysis and best practice recommendations. NSW public Heal Bull. 2011;22:78-83. 
401

20. Horak FB, Wrisley DM, Frank J. The Balance evaluation systems test (BESTest) to differentiate balance deficits. Phys Ther. 2009;89(5):484-498.

21. Messier SP, Royer TD, Craven TE. Long-Term Exercise and its Effect on Balance in Older, Osteoarthritic Adults : Results from the Fitness, Arthritis and Seniors trial (FAST). JAGS. 2000:131138.

22. Kannus $\mathrm{P}$, Sievänen $\mathrm{H}$, Palvanen $\mathrm{M}$, Järvinen $\mathrm{T}$, Parkkari J. Prevention of falls and consequent injuries in elderly people. 2005;366. doi:10.1016/\$0140-6736(05)67604-0

23. Kim J, Son J, Ko N, Yoon B. Unsupervised Virtual Reality-Based Exercise Program Improves Hip Muscle Strength and Balance Control in Older Adults: A Pilot Study. Arch Phys Med Rehabil. 2013;94(5):937-943. doi:10.1016/j.apmr.2012.12.010

24. Fry a C. The role of resistance exercise intensity on muscle fiber adaptation. Sport Med. 2004;34(10):663-669. doi:10.2165/00007256-200434100-00004

25. Mazzeo RS, Tanaka H. Exercise Prescription for the Elderly. Sport Med. 2001;31(11 LBMazzeo2001):809-818. doi:10.2165/00007256-200131110-00003

26. Correa C, LaRoche D, Cadore E, et al. 3 Different Types of Strength Training in Older Women. Int J Sports Med. 2012;33(12):962-969. doi:10.1055/s-0032-1312648

27. Pijnappels M, van der Burg JCE, Reeves ND, van Dieeen JH. Identification of elderly fallers by muscle strength measures. Eur J Appl Physiol. 2008;102(5):585-592. doi:http://dx.doi.org/10.1007/s00421-007-0613-6

28. Baggen RJ, Roie E Van, Dieën JH Van, Verschueren SM, Delecluse C. Weight bearing exercise can elicit similar peak muscle activation as medium - high intensity resistance exercise in elderly women. Eur J Appl Physiol. 2018;118(3):531-541. doi:10.1007/s00421-017-3793-8

29. Goto K, Ishii N, Kizuka T, Takamatsu K. The impact of metabolic stress on hormonal responses and muscular adaptations. Med Sci Sports Exerc. 2005;37(6):955-963. doi:10.1249/01.mss.0000170470.98084.39

30. Kan GAVAN, André E, Boirie Y, Onder G. CARLA TASK FORCE ON SARCOPENIA : PROPOSITIONS FOR CLINICAL TRIALS *. 2009;13(8). 
31. Glenn JM, Gray M, Binns A. The effects of loaded and unloaded high-velocity resistance training on functional fitness among community-dwelling older adults. Age Ageing. 2015;44(6):926-931. doi:10.1093/ageing/afv081

32. Diest M Van, Lamoth CJC, Stegenga J, Verkerke GJ, Postema K. Exergaming for balance training of elderly : state of the art and future developments. 2013.

33. Keshner EA. Virtual reality and physical rehabilitation : a new toy or a new research and rehabilitation tool ? 2004;2:1-2. doi:10.1186/1743-0003-1-8

34. Alkner A, Tesch PERA, Berg HE. Quadriceps EMG / force relationship in knee extension and leg press. 2000;(January 1999):459-463.

35. Onishi H, Yagi R, Akasaka K, Momose K, Ihashi K, Handa Y. Relationship between EMG signals and force in human vastus lateralis muscle using multiple bipolar wire electrodes. 2000;10:59-67.

36. Pijnappels M, Reeves ND, Maganaris CN, van Dieën JH. Tripping without falling; lower limb strength, a limitation for balance recovery and a target for training in the elderly. $J$ Electromyogr Kinesiol. 2008;18(2):188-196. doi:http://dx.doi.org/10.1016/j.jelekin.2007.06.004

37. Pandy MG, Lin Y-C, Kim HJ. Muscle coordination of mediolateral balance in normal walking. J Biomech. 2010;43(11):2055-2064. doi:10.1016/j.jbiomech.2010.04.010

38. Hermens H, Freriks B, Merletti R, et al. SENIAM. European recommendations for surface electromyography; 1999. Roessingh Res Dev Enschede.

39. De Luca CJ, Gilmore LD, Kuznetsov M, Roy SH. Filtering the surface EMG signal : Movement artifact and baseline noise contamination. J Biomchanics. 2010;43(8):1573-1579. doi:10.1016/j.jbiomech.2010.01.027

40. Konrad P. The abc of emg. A Pract Introd to Kinesiol Electromyogr. 2005;1:30-35.

41. Soltani P, Figueiredo P, Fernandes RJ, Vilas-boas JP. Muscle activation behavior in a swimming exergame : Di ff erences by experience and gaming velocity. Physiol Behav. 2017;181(August):2328. doi:10.1016/j.physbeh.2017.09.001

42. Fernandes Da Silva F, Aparecido de Souza R, Fernandes Dias E, Silveira L, Balbin Villaverde A. Effects of feedback on activation of the quadriceps during weight-bearing tasks of the Wii. J Phys 
Ther Sci. 2015;27:1701-1704.

456

43. Vries AW De, Faber G, Jonkers I, Dieen JH Van, Verschueren SMP. Virtual reality balance training for elderly : Similar skiing games elicit different challenges in balance training. Gait Posture. 2018;59(December 2016):111-116. doi:10.1016/j.gaitpost.2017.10.006

44. Vijver I Van De, Ridderinkhof KR, Wit S De. Age-related changes in deterministic learning from positive versus negative performance feedback. Aging, Neuropsychol Cogn. 2015;22(5):595-619. doi:10.1080/13825585.2015.1020917

45. Reed AE, Carstensen LL. The Theory Behind the Age-Related Positivity Effect. Front Psychol. 2012;3:339. doi:10.3389/fpsyg.2012.00339

46. Prata MG, Scheicher ME. Effects of strength and balance training on the mobility, fear of falling and grip strength of elderly female fallers. J Bodyw Mov Ther. 2015;19(4):646-650. doi:10.1016/j.jbmt.2014.11.013

47. Bisson E, Contant B, Sveistrup H. Functional Balance and Dual-Task Reaction Times in Older Adults Are Improved by Virtual Reality and Biofeedback Training. 2007;10(1):16-23. doi:10.1089/cpb.2006.9997

48. Pedroli E, Id LG, Serino S, et al. System Combining Cognitive and Physical Therapy in a Virtual Environment : Positive Bike. 2018. doi:10.3390/s18072343

49. Willaert J, De Vries A, Tavernier J, Van Dieen J, Jonkers I, Verschueren Sa. Does a novel exergame challenge balance and activate muscles more than existing off-the-shelf exergames? J Neuroengineering Rehabil., submitted. 


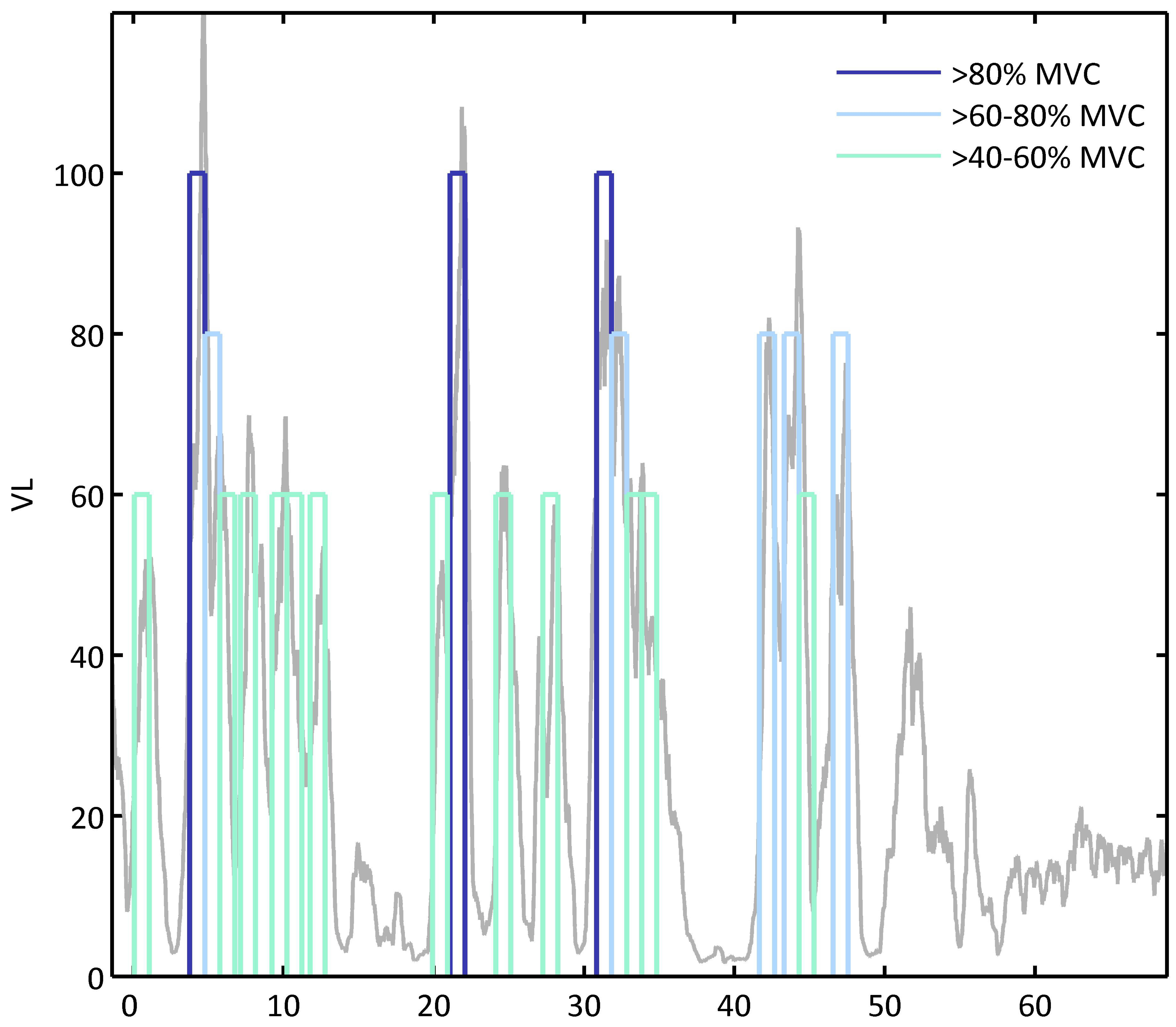




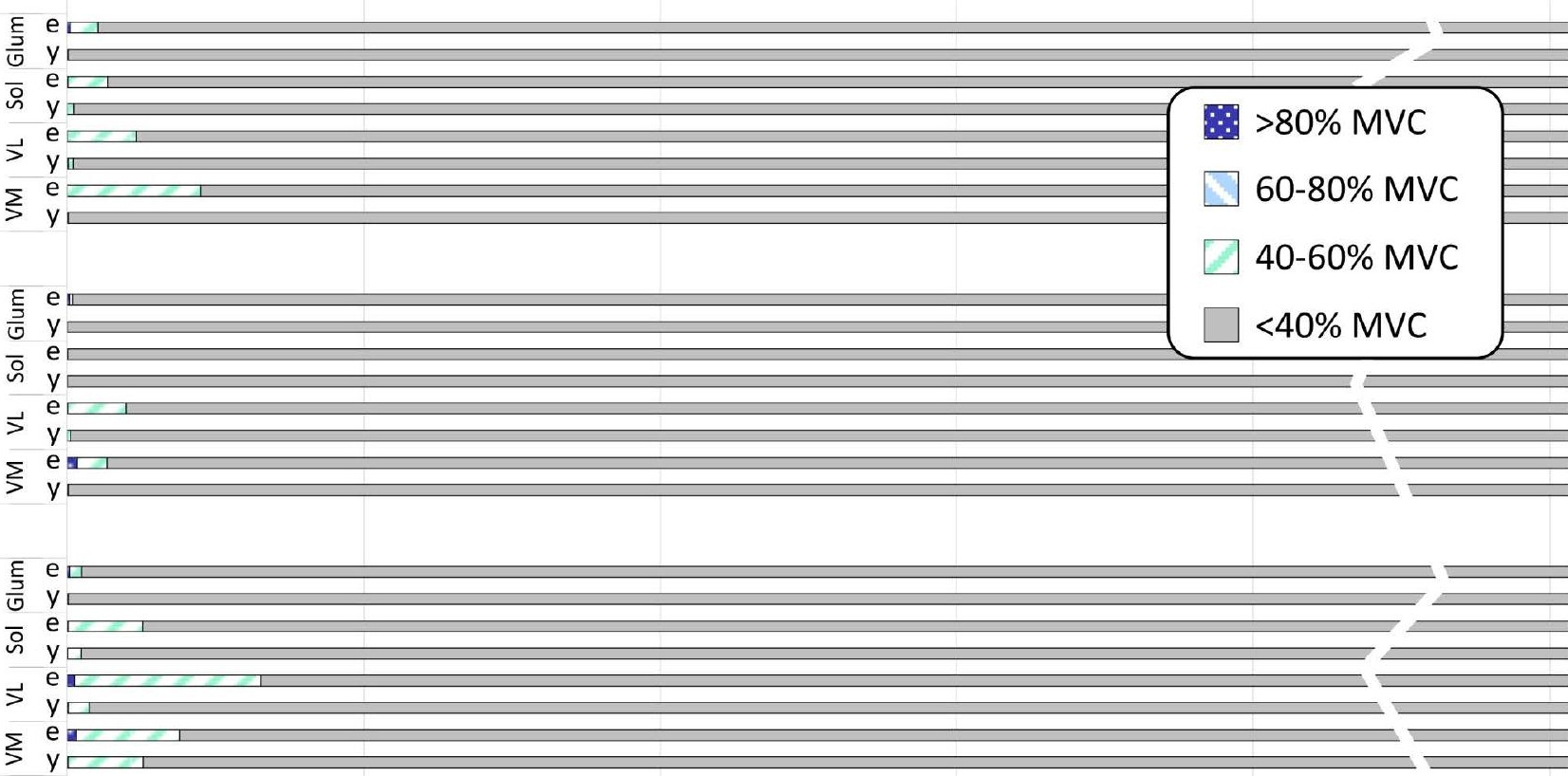

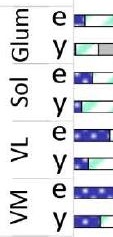

艼 $\mathrm{e}$

- e a

i $y$...

$\supset \mathrm{e}$

$\sum \mathrm{e}$

$y=$

乌 $\mathrm{e}$ ㄷ

으 $\mathrm{y}$

$>\quad \mathrm{e}$

$\sum \begin{aligned} & e \\ & y\end{aligned}$

VI I

a

E $\mathrm{e} \sin$

근 $\mathrm{y}$

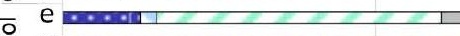

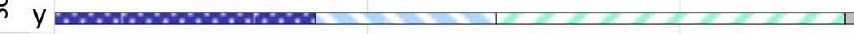

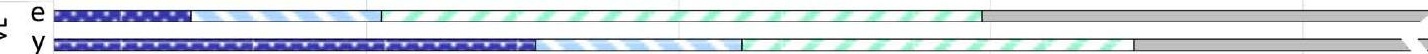

$\sum \mathrm{e}$ mawam

\begin{tabular}{ccccccc}
\hline $0 \%$ & $5 \%$ & $10 \%$ & $15 \%$ & $20 \%$ & $100 \%$
\end{tabular} 

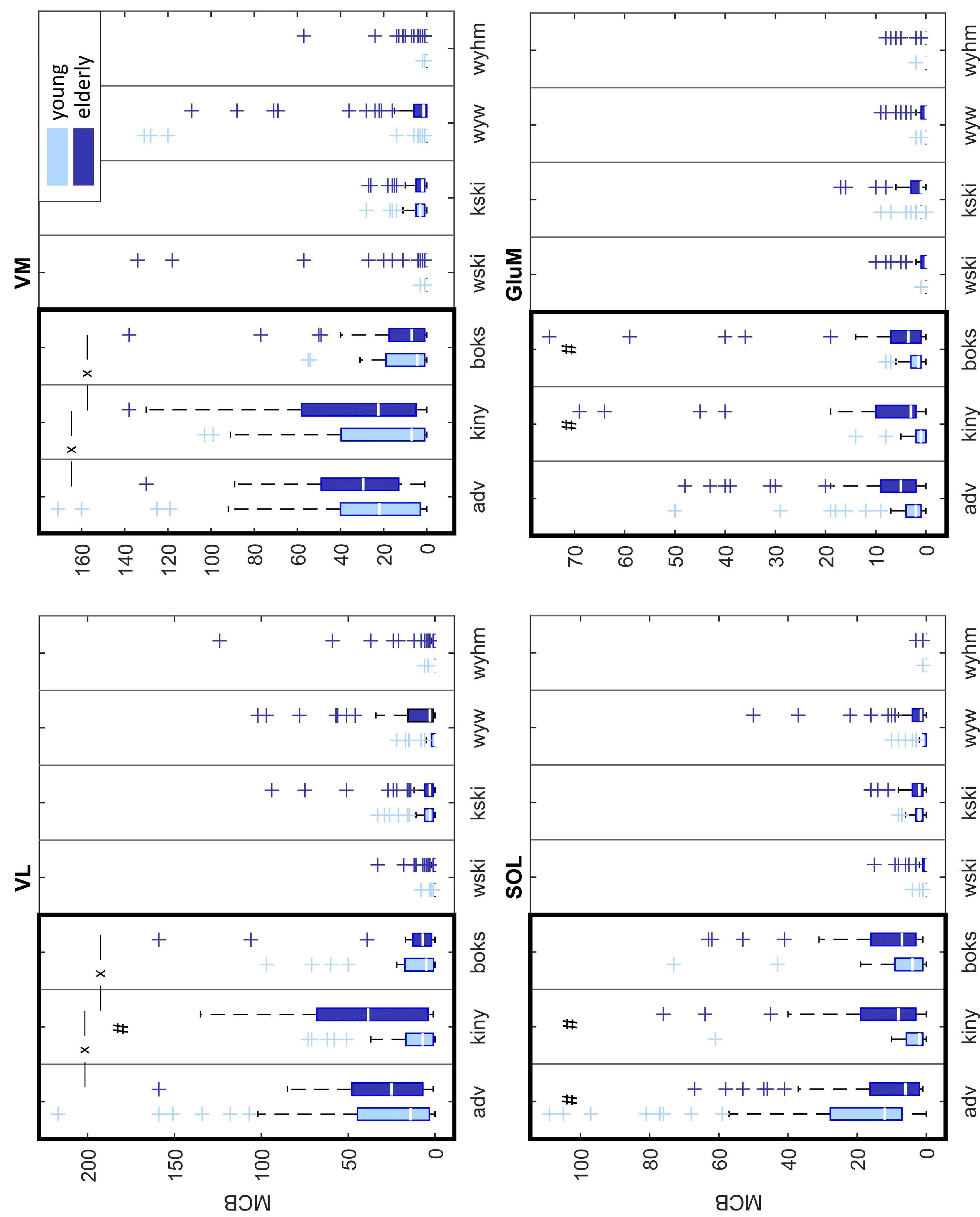